\title{
Chemical Abundance Analysis of Tucana III, the Second $r$-process Enhanced Ultra-faint Dwarf Galaxy*
}

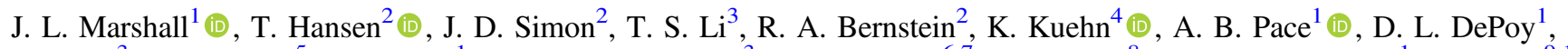

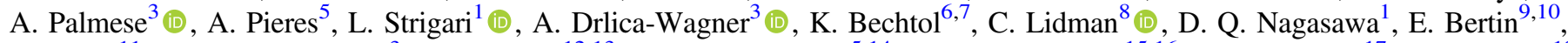
D. Brooks ${ }^{11}$ (D) E. Buckley-Geer ${ }^{3}$, D. L. Burke ${ }^{12,13}$, A. Carnero Rosell ${ }^{5,14}$, M. Carrasco Kind ${ }^{15,16}{ }_{\mathbb{D}}$, J. Carretero ${ }^{17}$, C. E. Cunha ${ }^{12}$, C. B. D’Andrea ${ }^{18}$, L. N. da Costa ${ }^{5,19}$, J. De Vicente ${ }^{14}$, S. Desai ${ }^{20}$, P. Doel ${ }^{11}$, T. F. Eifler $^{21,22}$, B. Flaugher ${ }^{3}$, P. Fosalba ${ }^{23,24}$,

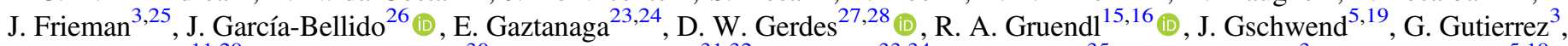
W. G. Hartley ${ }^{11,29}$, D. L. Hollowood ${ }^{30}$, K. Honscheid ${ }^{31,32}$, B. Hoyle ${ }^{33,34}$, D. J. James ${ }^{35}$, N. Kuropatkin ${ }^{3}$, M. A. G. Maia ${ }^{5,19}$, F. Menanteau ${ }^{15,16}$, C. J. Miller ${ }^{27,28}$, R. Miquel ${ }^{17,36}$ (ㅁ) A. A. Plazas ${ }^{22}$, E. Sanchez ${ }^{14}$, B. Santiago ${ }^{5,37}$, V. Scarpine ${ }^{3}$, M. Schubnell ${ }^{28}$, S. Serrano ${ }^{23,24}$, I. Sevilla-Noarbe ${ }^{14}$, M. Smith ${ }^{38}$, M. Soares-Santos ${ }^{39}$ (i), E. Suchyta ${ }^{40}$, M. E. C. Swanson ${ }^{16}$, G. Tarle ${ }^{28}$ (1) and W. Wester ${ }^{3}$

(DES Collaboration)

${ }^{1}$ Mitchell Institute for Fundamental Physics and Astronomy and Department of Physics and Astronomy, Texas A\&M University, College Station, TX 77843-4242,

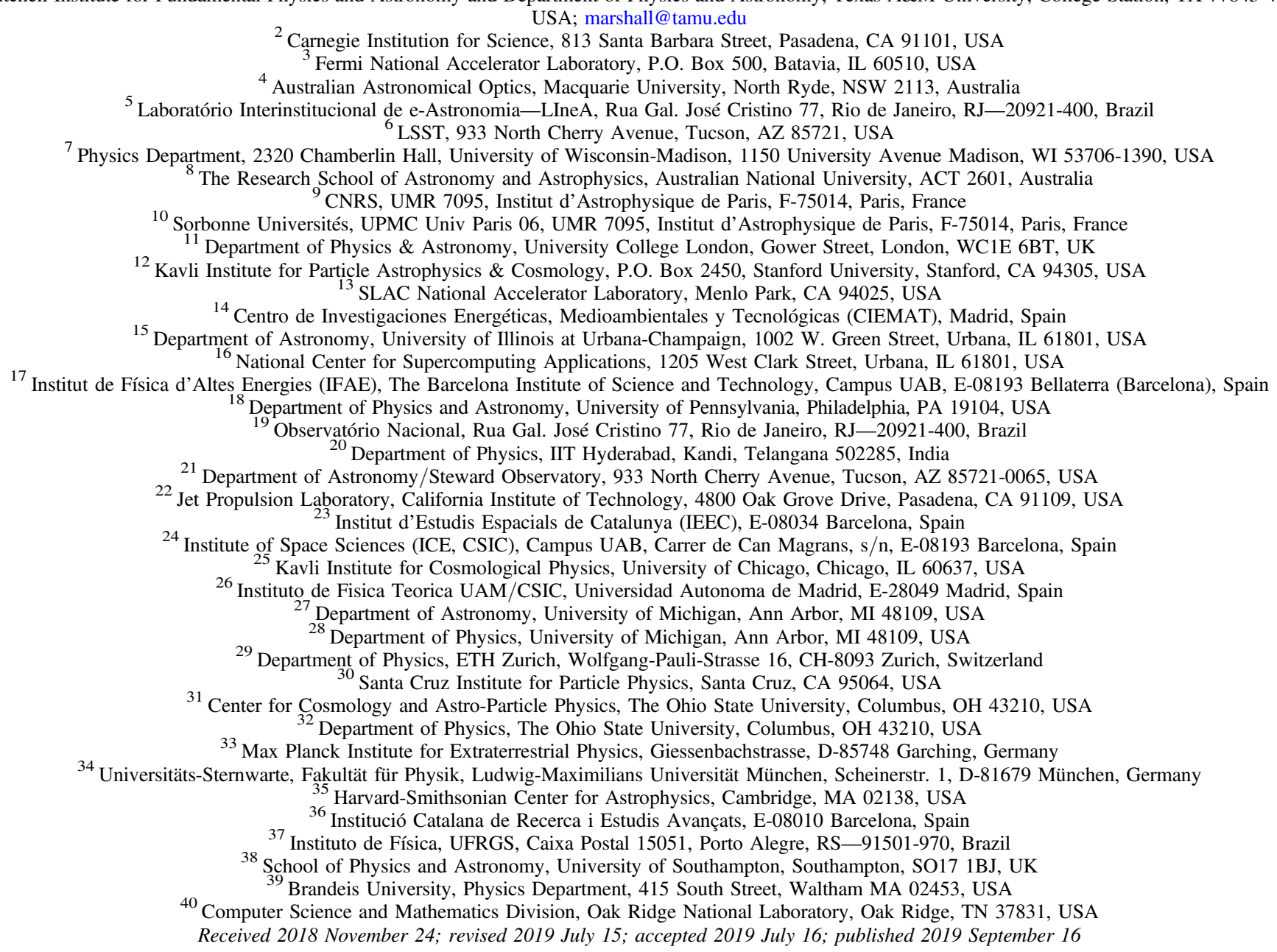

\begin{abstract}
We present a chemical abundance analysis of four additional confirmed member stars of Tucana III, a Milky Way satellite galaxy candidate in the process of being tidally disrupted as it is accreted by the Galaxy. Two of these stars are centrally located in the core of the galaxy while the other two stars are located in the eastern and western tidal tails. The four stars have chemical abundance patterns consistent with the one previously studied star in Tucana III: they are moderately enhanced in $r$-process elements, i.e., they have $\langle[\mathrm{Eu} / \mathrm{Fe}]\rangle \approx+0.4 \mathrm{dex}$.
\end{abstract}

\footnotetext{
* This paper includes data gathered with the $6.5 \mathrm{~m}$ Magellan Telescopes located at Las Campanas Observatory, Chile.
} 
The non-neutron-capture elements generally follow trends seen in other dwarf galaxies, including a metallicity range of $0.44 \mathrm{dex}$ and the expected trend in $\alpha$-elements, i.e., the lower metallicity stars have higher $\mathrm{Ca}$ and $\mathrm{Ti}$ abundances. Overall, the chemical abundance patterns of these stars suggest that Tucana III was an ultra-faint dwarf galaxy, and not a globular cluster, before being tidally disturbed. As is the case for the one other galaxy dominated by $r$-process enhanced stars, Reticulum II, Tucana III's stellar chemical abundances are consistent with pollution from ejecta produced by a binary neutron star merger, although a different $r$-process element or dilution gas mass is required to explain the abundances in these two galaxies if a neutron star merger is the sole source of $r$-process enhancement.

Unified Astronomy Thesaurus concepts: Chemical abundances (224); Chemically peculiar stars (226); Dwarf galaxies (416)

Supporting material: machine-readable table

\section{Introduction}

Over sixty years ago, Burbidge et al. (1957) summarized a plausible story for the nucleosynthesis of every element in the Periodic Table. Since that time, observations of the production processes of all but the heaviest elements have confirmed early theories, with only the production site (or sites) of the rapid neutron-capture, or $r$-process, elements eluding direct observation. The recent detection of a binary neutron star merger event enabled by LIGO (Abbott et al. 2017) and extensive efforts to follow up the event (e.g., Drout et al. 2017; Shappee et al. 2017) have added a further dimension to the study of $r$-process element production, perhaps enabling the direct observation of the production sites of the heaviest elements for the first time.

In the Milky Way halo and in dwarf galaxies, stars have been found showing large enhancements in $r$-process elements. These are divided into two subclasses: moderately enhanced $r$-I stars $(+0.3<[\mathrm{Eu} / \mathrm{Fe}]<+1.0)$ and highly enhanced $r$-II stars $([\mathrm{Eu} / \mathrm{Fe}]>+1.0)$ (Beers \& Christlieb 2005). These stars, which are often metal-poor, are quite rare, and as of a few years ago, only $\sim 100 r$-I and $20 r$-II stars were known, nearly all located in the halo. Only recently have $r$-process enhanced stars begun to be found in larger numbers via dedicated searches (e.g., Barklem et al. 2005; Hansen et al. 2018; Sakari et al. 2018a) or in serendipitous discoveries during chemical study of Milky Way satellite galaxies (Ji et al. 2016a; Roederer et al. 2016; Hansen et al. 2017).

Interestingly, two recently discovered Milky Way satellite galaxies, Reticulum II and Tucana III, have been shown to be enhanced in $r$-process elements. These discoveries have been enabled by modern deep, wide-field imaging surveys such as the Dark Energy Survey (DES; DES Collaboration 2005), Magellanic Satellites Survey (MagLiteS; Drlica-Wagner et al. 2016), Survey of the Magellanic Stellar History (SMASH; Martin et al. 2015), and Pan-STARRS (Chambers et al. 2016), which have revealed faint, previously unknown stellar associations. Of particular interest are the discoveries of many dark matter-dominated ultra-faint dwarf galaxies and tidally disrupted stellar streams that have been found in and around the Milky Way halo using images from DES (Bechtol et al. 2015; Drlica-Wagner et al. 2015; Kim \& Jerjen 2015; Kim et al. 2015; Koposov et al. 2015; Luque et al. 2016, 2017; Shipp et al. 2018).

The first of the DES-discovered ultra-faint dwarf galaxies to be kinematically confirmed as a dark matter-dominated ultrafaint dwarf galaxy was Reticulum II (Ret II; Simon et al. 2015; Walker et al. 2015). The nine brightest confirmed member stars were subsequently chemically analyzed by Ji et al. (2016a, 2016c) and Roederer et al. (2016); these authors showed that most of the stars in Ret II are strongly enhanced in the $r$-process elements, i.e., they are $r$-II stars. Since $r$-II stars are so rare in the Milky Way, it was particularly notable to have found a galaxy seemingly composed primarily of these types of stars. Even more interesting, the authors conclude that the high fraction of $r$-process enhanced stars must be due to Ret II's chemical history being dominated by a single nucleosynthetic event, most likely a binary neutron star merger.

A second ultra-faint dwarf galaxy, Tucana III (Tuc III), the subject of this paper, has since been shown to be enhanced in $r$-process elements as well, although to a lower level of enhancement than Ret II. Tuc III was first identified as a candidate Milky Way satellite galaxy in the DES Year 2 data set (Drlica-Wagner et al. 2015). Simon et al. (2017) measured radial velocities of candidate member stars in Tuc III and used 26 confirmed member stars to show that, if Tuc III is a galaxy, it may be the Milky Way satellite galaxy with the lowest mass and velocity dispersion and also the smallest metallicity dispersion of any known dwarf galaxy (Segue 2 has a similarly low mass Kirby et al. 2013). Despite the fact that these characteristics place Tuc III in a part of parameter space where globular clusters and dwarf galaxies cannot be cleanly separated, Simon et al. (2017) concluded that Tuc III is most likely a dwarf galaxy and not a globular cluster. A possible explanation for Tuc III's low metallicity and large size is that the prominent central overdensity is actually the center of a previously more populous galaxy that has been tidally stripped, leaving only the core of the galaxy intact. The brightest confirmed member star in Tuc III has been chemically analyzed by Hansen et al. (2017), who classified it as an $r$-I star.

Tuc III is unique among recently discovered candidate Milky Way satellites in that the DES discovery images show a linear structure in the filtered stellar density map that extends two degrees to either side of the central overdensity. The papers reporting the discovery (Drlica-Wagner et al. 2015) and kinematic confirmation (Simon et al. 2017) of Tuc III suggested that this feature may be consistent with a set of leading and trailing tails resulting from tidal disruption as Tuc III merges with the Milky Way halo. Indeed, Li et al. (2018a) have recently confirmed that these structures are kinematically associated with the Tuc III system, adding 22 confirmed members of the Tuc III tidal tails to the 26 central core stars confirmed by Simon et al. (2017). The tidal tails extend at least $2^{\circ}$ to either side of the core and show a significant velocity gradient across the structure, as expected for a system being tidally disrupted as it merges with the Milky Way. Furthermore, Erkal et al. (2018) used these same stars and their measured velocity gradient along with predicted space velocities to fit an orbit about the Milky Way, indicating that 


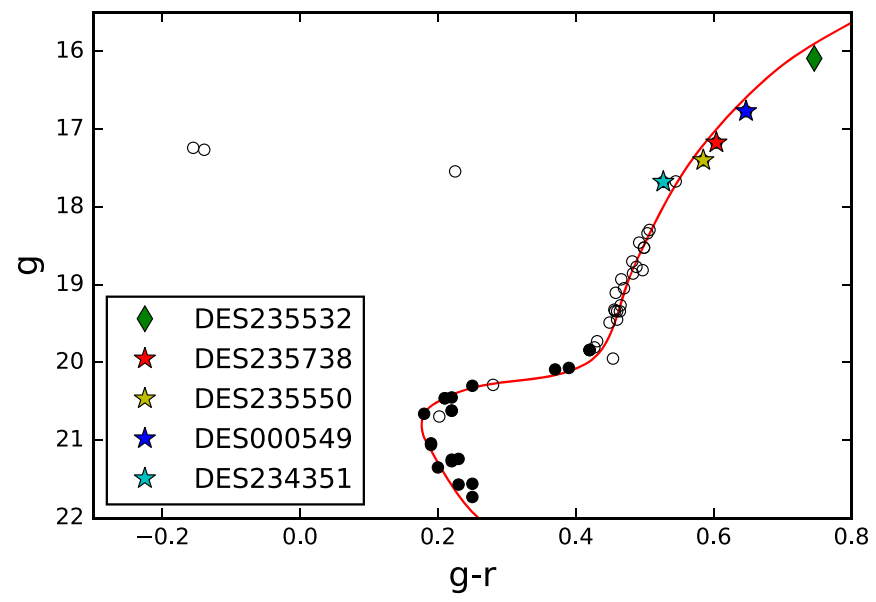

Figure 1. Color-magnitude diagram composed with DES photometry for confirmed member stars of Tuc III. DES J235532 was shown to be an $r$-I star by Hansen et al. (2017) and is marked with a green diamond; the four stars studied in this work are marked with stars: the red and yellow stars are located in the core of the galaxy; the blue and magenta stars are in the tails. Filled circles mark other confirmed member stars in the core of Tuc III from Simon et al. (2017); open circles are confirmed member stars located in the core and tidal tails from Li et al. (2018a). A Dartmouth isochrone (Dotter et al. 2008) of a stellar population having an age of $12.5 \mathrm{Gyr},[\mathrm{Fe} / \mathrm{H}]=-2.3,[\alpha / \mathrm{Fe}]=0.2$ and a distance of $25 \mathrm{kpc}$ is overplotted (red curve).

Tuc III has had a recent close passage with the Large Magellanic Cloud. This prediction was further refined with Gaia proper motions (Simon 2018), which demonstrate that Tuc III is now on a highly eccentric orbit around the Milky Way with a pericenter of $\sim 3 \mathrm{kpc}$.

In this paper we present the chemical abundance analysis of four additional stars in the Tuc III stellar system; two located in the core of the galaxy and two in the tidal tails. This paper is organized as follows: In Section 2 we describe the observations of the four stars. We describe the radial velocity and abundance measurements of these stars in Section 3 and present the results of these measurements in Section 4. In Section 5 we discuss the implications of the chemical abundance patterns of the Tuc III member stars and in Section 6 we conclude.

\section{Observations and Data Reduction}

We selected a sample of four confirmed member stars of Tuc III that had not been studied previously with high resolution spectroscopy: two stars were selected from the sample of confirmed member stars of Simon et al. (2017), located in the core of the galaxy, and two stars were selected in Tuc III's tidal tails from the sample of confirmed member stars of Li et al. (2018a). Throughout this work we include one star previously chemically analyzed by Hansen et al. (2017) for reference.

A color-magnitude diagram of the Tuc III member stars is presented in Figure 1, with reddening-corrected stellar magnitudes from Li et al. (2018a) and Simon et al. (2017). Figure 2 shows the locations of these five stars with respect to confirmed member stars in Tuc III's core (Simon et al. 2017) and tail (Li et al. 2018a). In Figures 1 and 2, astrometry and photometry are those reported by $\mathrm{Li}$ et al. (2018a) when available, because that more recent work presents measurements from the DES DR1 public data release (DES Collaboration 2018), an updated, better-calibrated version of the DES catalog than the Y2Q1 catalog (Drlica-Wagner et al. 2015) used by Simon et al. (2017).
Observations were performed with the MIKE spectrograph (Bernstein et al. 2003) at the Magellan-Clay Telescope at Las Campanas Observatory. Observations took place on 2016 August 5-7. We used a 0 . 7 slit with $2 \times 2$ pixel binning to obtain a spectral resolution of $R=\lambda / \Delta \lambda \approx 41,000$ in the blue and 32,000 in the red. The spectra cover $3310 \AA<\lambda<5000 \AA$ in the blue channel and $4830 \AA<\lambda<9160 \AA$ in the red.

Conditions were somewhat marginal on the first two nights, with some cloud cover and seeing of $0 . " 8-1 . " 2$; clouds cleared and seeing improved to $0 . " 7$ on the third night. Each star was observed on only one night, with multiple 30 minute integrations interspersed with ThAr comparison lamp spectra at intervals of no more than one hour to facilitate precise wavelength calibration and radial velocity measurements. In addition to the program stars, at least one radial velocity standard star was observed on each night; telluric standards were observed on the first and third nights.

An observing log is given in Table 1. The four stars studied here will be referred to as DES J235738, DES J235550, DES J000549, and DES J234351 for brevity. We include the star DES J235532 studied by Hansen et al. (2017) for reference. Also included in Table 1 are the DES astrometry and $g$ and $g-r$ photometry for each star reported by Li et al. (2018a).

Reduction of the data, including bias subtraction, flatfielding, spectral extraction, wavelength calibration, and coadding was completed on the mountain with the latest version of the MIKE pipeline (Kelson 2003). Formal signal-tonoise ratios (S/Ns) were measured at 4100 and $5500 \AA$ using the IRAF task splot and are presented in Table 1.

\section{Stellar Parameter Determination and Chemical Abundance Analysis}

\subsection{Radial Velocities}

Radial velocities for each star were measured by comparing the program star with a radial velocity standard star (HD 136202) observed on the first night of the run. Radial velocities were derived via cross-correlation of each order of the program star spectrum with the corresponding order of the standard star spectrum. The blue and the red arms of the spectrograph were considered independently. The mean values of the resulting relative velocities from each arm, for 28 orders in the blue and 6 orders in the red, with $3 \sigma$ outliers rejected, e.g., $\mathrm{RV}_{\text {blue }}=\sum\left(v_{n \text {,blue }}\right) / N$, where $v_{n \text {,blue }}$ is the velocity derived from each order of the blue arm and $N$ is the total number of blue orders. These velocities were then averaged to form the final reported velocity for each star: $R V_{\text {final }}=\left(R V_{\text {blue }}+R V_{\text {red }}\right) / 2$. Errors were derived using the standard deviation of the individual velocities determined from each order, again considering the blue and red arms separately. The reported error on each velocity is calculated as $\sigma_{\mathrm{RV}}$ final $=\left(\sigma_{\mathrm{RV}_{\text {blue }}}^{2} / 2+\sigma_{\mathrm{RV}}^{2} / 2\right)^{1 / 2}$. Measured radial velocities for all four stars are presented in Table 2 . The radial velocity measurements were used to place each program star spectrum on a wavelength scale associated with rest wavelengths. These velocities can also be used to investigate whether the stars are in fact binaries, as discussed in Section 5.3.

\subsection{Stellar Parameter Measurements and Abundance Analysis}

Stellar parameters for the four stars were derived spectroscopically, following the method described by Hansen et al. (2017). In brief, we used the 2017 version of the MOOG spectral synthesis program (Sneden 1973), making the 
Table 1

Observing Log

\begin{tabular}{|c|c|c|c|c|c|c|c|c|c|}
\hline Object Name & $\begin{array}{l}\text { R.A. }^{\mathrm{a}} \\
(\mathrm{J} 2000)\end{array}$ & $\begin{array}{l}\text { Decl. }^{\mathrm{a}} \\
(\mathrm{J} 2000)\end{array}$ & $\begin{array}{c}g^{\mathrm{a}} \\
(\mathrm{mag})\end{array}$ & $\begin{array}{l}g-r^{\mathrm{a}} \\
(\mathrm{mag})\end{array}$ & $\begin{array}{l}\text { Date Observed } \\
\text { (MJD) }\end{array}$ & $\begin{array}{l}t_{\text {exp }} \\
(\mathrm{hr})\end{array}$ & $\begin{array}{c}\mathrm{S} / \mathrm{N} \\
\text { at } 4200 \AA\end{array}$ & $\begin{array}{c}\mathrm{S} / \mathrm{N} \\
\text { at } 5500 \AA\end{array}$ & Location \\
\hline DES J235532-593115 & $23: 55: 32.7$ & $-59: 31: 15.0$ & 16.090 & 0.746 & 57248 & 3.5 & 20 & 50 & Core \\
\hline DES J235550-593300 & $23: 55: 49.9$ & $-59: 33: 00.0$ & 17.400 & 0.585 & 57607.77 & 2 & 20 & 30 & Core \\
\hline DES J000549-593406 & 00:05:48.7 & $-59: 34: 06.1$ & 16.770 & 0.647 & 57605.78 & 4.5 & 30 & 40 & Tail \\
\hline DES J234351-593926 & $23: 43: 50.8$ & $-59: 39: 25.6$ & 17.678 & 0.527 & 57606.82 & 2.5 & 10 & 20 & Tail \\
\hline
\end{tabular}

Notes.

${ }^{a}$ Astrometry and dereddened photometry from Li et al. (2018a).

${ }^{\mathrm{b}}$ Reported at the midpoint of the observation.

${ }^{c}$ From Hansen et al. (2017), included for reference.

Table 2

Measured Stellar Parameters

\begin{tabular}{|c|c|c|c|c|c|}
\hline ID & $\begin{array}{c}v_{\text {hel }} \\
\left(\mathrm{km} \mathrm{s}^{-1}\right)\end{array}$ & $\begin{array}{l}T_{\text {eff }} \\
(\mathrm{K})\end{array}$ & $\log g$ & $\begin{array}{c}v_{\text {micro }} \\
\left(\mathrm{km} \mathrm{s}^{-1}\right)\end{array}$ & {$[\mathrm{Fe} / \mathrm{H}]$} \\
\hline DES J235532 & $-103.4 \pm 0.3$ & $4720 \pm 100$ & $1.33 \pm 0.3$ & $2.0 \pm 0.3$ & $-2.25 \pm 0.18$ \\
\hline DES J235550 & $-102.9 \pm 0.8$ & $4720 \pm 150$ & $1.55 \pm 0.3$ & $1.5 \pm 0.3$ & $-2.69 \pm 0.17$ \\
\hline DES J000549 & $-92.6 \pm 0.9$ & $4675 \pm 150$ & $1.39 \pm 0.3$ & $1.7 \pm 0.3$ & $-2.61 \pm 0.08$ \\
\hline DES J234351 & $-121.5 \pm 1.1$ & $4900 \pm 150$ & $1.88 \pm 0.3$ & $1.6 \pm 0.3$ & $-2.69 \pm 0.14$ \\
\hline
\end{tabular}

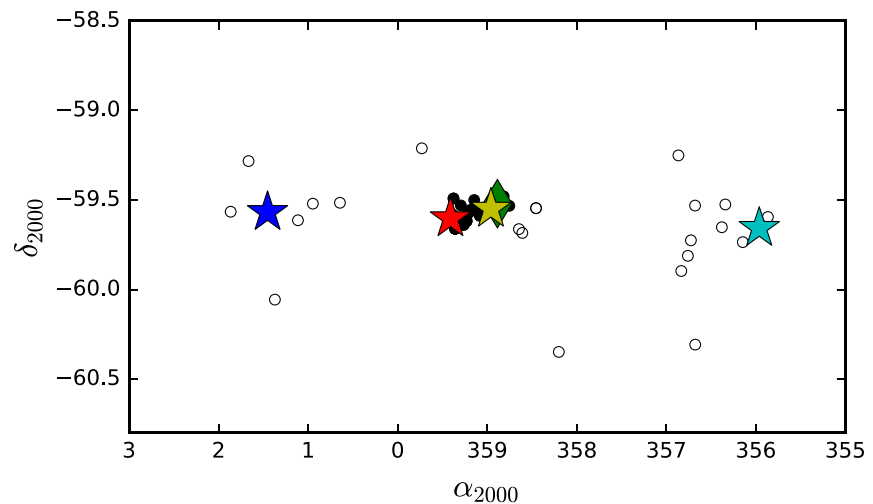

Figure 2. Angular distribution of confirmed member stars in the Tuc III core and tidal tails. Symbols are the same as those in Figure 1.

assumption of local thermodynamic equilibrium and including Rayleigh scattering treatment as described by Sobeck et al. (2011). ${ }^{41}$ Initial effective temperatures were determined from excitation equilibrium of $\mathrm{Fe} \mathrm{I}$ lines and thereafter placed on a photometric scale using the relation from Frebel et al. (2013). Following this the surface gravities $(\log g$ ) were determined from ionization equilibrium between the Fe I and Fe II lines. Finally microturbulent velocities $(\xi)$ were determined by removing any trend in line abundances with reduced equivalent widths for the $\mathrm{Fe}$ I lines. For the four stars studied here, J235738, J235550, J000549, and J234351, we were able to use 103, 122, 157, and $90 \mathrm{Fe} \mathrm{I}$ lines and 15, 13, 20, and $16 \mathrm{Fe}$ II lines, respectively, for this analysis. Final stellar parameters are presented in Table 2 and the lines used for the analysis of each star are listed in Table 3.

\footnotetext{
$\overline{41}$ https://github.com/alexji/moog 17 scat
}

Abundances were derived from equivalent width measurements and spectral synthesis using MOOG. We used $\alpha$-enhanced $([\alpha / \mathrm{Fe}]=+0.4)$ 1D LTE ATLAS9 model atmospheres (Castelli \& Kurucz 2003) and the solar photosphere abundances from Asplund et al. (2009). Line lists were generated using the linemake package ${ }^{42}$ (C. Sneden 2019, private communication), including molecular lines for $\mathrm{CH}, \mathrm{C}_{2}$, and $\mathrm{CN}$ and isotopic shift and hyperfine structure information. Measured stellar abundances are presented in Table 4.

We note here that the effective temperature derived for three of these stars (including J235532) are all equal, which is unexpected given that $\mathbf{J} 235532$ is somewhat redder than the other two stars. We have carefully reconsidered the derived effective temperatures for all five stars, including J235532, and find no errors in the analysis. Expected values from the Dartmouth isochrone (shown in Figure 1) suggest a temperature difference of $\Delta T \sim 300 \mathrm{~K}$ between stars having the colors of J235532 and J235550, which agrees with our derived spectroscopic temperatures within errors.

Uncertainties on the derived abundance for J000549 arising from stellar parameter uncertainties are listed in Table 5. These uncertainties were computed by deriving abundances with different atmospheric models, each with one parameter varied by its uncertainty as given in Table 2 and added in quadrature. As all stars have similar stellar parameters and spectral quality we consider these uncertainties to be applicable to all four stars studied here.

Sample synthetic spectra for absorption lines of $\mathrm{Sr}, \mathrm{Ba}$, and Eu can be found in Figure 3, overlaid onto the observed spectra.

\footnotetext{
${ }^{42}$ https://github.com/vmplacco/linemake
} 
Table 3

$\mathrm{Fe} \mathrm{I}$ and Fe II Lines Used for Parameter Determination

\begin{tabular}{|c|c|c|c|c|c|c|}
\hline Stellar ID & Species & $\begin{array}{c}\lambda \\
(\stackrel{\AA}{)})\end{array}$ & $\begin{array}{c}\chi \\
(\mathrm{eV})\end{array}$ & $\log g f$ & $\begin{array}{c}\text { EW } \\
(\mathrm{m} \AA)\end{array}$ & $\log \epsilon$ \\
\hline J000549-593406 & $\mathrm{Fe} \mathrm{I}$ & 3765.54 & 3.240 & 0.480 & 78.63 & 4.638 \\
\hline J000549-593406 & $\mathrm{Fe} \mathrm{I}$ & 3790.09 & 0.989 & -1.740 & 106.00 & 4.988 \\
\hline J000549-593406 & $\mathrm{Fe} \mathrm{I}$ & 3807.54 & 2.221 & -0.990 & 80.07 & 4.934 \\
\hline J000549-593406 & $\mathrm{Fe} \mathrm{I}$ & 3876.04 & 1.010 & -2.890 & 74.29 & 5.158 \\
\hline J000549-593406 & $\mathrm{Fe} \mathrm{I}$ & 3917.18 & 0.989 & -2.150 & 101.40 & 5.122 \\
\hline J000549-593406 & Fe II & 4233.17 & 2.580 & -1.810 & 81.79 & 4.746 \\
\hline J000549-593406 & $\mathrm{Fe}$ II & 4416.83 & 2.780 & -2.410 & 57.10 & 4.979 \\
\hline J000549-593406 & $\mathrm{Fe}$ II & 4489.18 & 2.828 & -2.971 & 22.98 & 4.869 \\
\hline J000549-593406 & $\mathrm{Fe}$ II & 4491.41 & 2.850 & -2.640 & 28.78 & 4.702 \\
\hline
\end{tabular}

(This table is available in its entirety in machine-readable form.)

Table 4

Measured Abundances

\begin{tabular}{|c|c|c|c|c|c|c|c|c|c|c|c|c|c|c|c|c|}
\hline \multirow[b]{2}{*}{ Element } & \multicolumn{4}{|c|}{ J000549 } & \multicolumn{4}{|c|}{$\mathrm{J} 234351$} & \multicolumn{4}{|c|}{$\mathrm{J} 235550$} & \multicolumn{4}{|c|}{$\mathrm{J} 235738$} \\
\hline & {$[\mathrm{X} / \mathrm{Fe}]$} & $\log \epsilon(\mathrm{X})$ & $\sigma$ & $N$ & {$[\mathrm{X} / \mathrm{Fe}]$} & $\log \epsilon(\mathrm{X})$ & $\sigma$ & $\bar{N}$ & {$[\mathrm{X} / \mathrm{Fe}]$} & $\log \epsilon(\mathrm{X})$ & $\sigma$ & $N$ & {$[\mathrm{X} / \mathrm{Fe}]$} & $\log \epsilon(\mathrm{X})$ & $\sigma$ & $N$ \\
\hline $\mathrm{C}$ & -0.43 & 5.39 & $\cdots$ & & -0.28 & 5.46 & $\ldots$ & & -0.19 & 5.55 & $\cdots$ & & -0.05 & 5.80 & $\cdots$ & \\
\hline $\mathrm{N}$ & $<2.00$ & $<7.22$ & $\cdots$ & & $<1.5$ & $<6.64$ & $\ldots$ & & $<1.0$ & $<6.14$ & $\cdots$ & & $<0.5$ & $<5.75$ & $\cdots$ & \\
\hline $\mathrm{NaI}$ & $\ldots$ & $\ldots$ & $\cdots$ & & 0.32 & 3.87 & 0.35 & 2 & $\cdots$ & $\cdots$ & $\cdots$ & & 0.53 & 4.19 & 0.33 & 2 \\
\hline MgI & 0.45 & 5.44 & 0.29 & 9 & 0.50 & 5.41 & 0.37 & 7 & 0.40 & 5.31 & 0.27 & 8 & 0.64 & 5.66 & 0.31 & 5 \\
\hline AlI & -0.36 & 3.48 & 0.39 & 2 & -0.99 & 2.78 & 0.36 & 2 & $\cdots$ & $\cdots$ & $\cdots$ & 2 & -0.35 & 3.52 & $\cdots$ & 1 \\
\hline SiI & 0.39 & 5.29 & 0.33 & 2 & 0.18 & 5.00 & 0.33 & 2 & 0.39 & 5.21 & 0.31 & 2 & 0.00 & 4.93 & $\cdots$ & 1 \\
\hline KI & 0.71 & 3.13 & 0.25 & 2 & 0.41 & 2.75 & $\cdots$ & 1 & 0.71 & 3.05 & $\cdots$ & 1 & 0.56 & 3.01 & 0.31 & 2 \\
\hline $\mathrm{CaI}$ & 0.40 & 4.13 & 0.28 & 19 & 0.35 & 4.00 & 0.28 & 11 & 0.44 & 4.09 & 0.28 & 13 & 0.46 & 4.22 & 0.28 & 13 \\
\hline ScII & 0.15 & 0.69 & 0.28 & 5 & 0.09 & 0.55 & 0.27 & 4 & -0.01 & 0.45 & 0.28 & 6 & 0.05 & 0.62 & 0.30 & 6 \\
\hline TiI & 0.07 & 2.41 & 0.31 & 18 & 0.17 & 2.43 & 0.34 & 7 & 0.15 & 2.41 & 0.36 & 12 & 0.28 & 2.65 & 0.32 & 8 \\
\hline TilI & 0.46 & 2.80 & 0.29 & 39 & 0.31 & 2.57 & 0.29 & 11 & 0.50 & 2.76 & 0.31 & 32 & 0.41 & 2.78 & 0.32 & 26 \\
\hline VI & -0.16 & 1.16 & $\ldots$ & 1 & 0.06 & 1.30 & $\ldots$ & 1 & -0.18 & 6.44 & $\ldots$ & 1 & -0.13 & 1.22 & $\ldots$ & 1 \\
\hline VII & 0.07 & 1.39 & 0.23 & 2 & 0.13 & 1.37 & 0.21 & 2 & 0.10 & 6.72 & 0.25 & 2 & 0.00 & 1.35 & $\cdots$ & 1 \\
\hline $\mathrm{CrI}$ & -0.38 & 2.65 & 0.32 & 14 & -0.34 & 2.61 & 0.33 & 7 & -0.26 & 2.69 & 0.34 & 9 & -0.17 & 2.89 & 0.34 & 6 \\
\hline CrII & 0.08 & 3.11 & 0.23 & 2 & 0.29 & 3.24 & 0.30 & 2 & 0.35 & 3.30 & 0.20 & 2 & 0.24 & 3.30 & $\ldots$ & 1 \\
\hline MnI & -0.50 & 2.32 & 0.24 & 6 & -0.42 & 2.32 & 0.25 & 5 & -0.50 & 2.25 & 0.23 & 6 & -0.39 & 2.46 & 0.23 & 4 \\
\hline $\mathrm{FeI}$ & -2.61 & 4.89 & 0.17 & 157 & -2.69 & 4.81 & 0.20 & 90 & -2.69 & 4.81 & 0.21 & 122 & -2.58 & 4.92 & 0.20 & 103 \\
\hline FeII & -2.62 & 4.88 & 0.18 & 20 & -2.70 & 4.80 & 0.17 & 16 & -2.69 & 4.81 & 0.08 & 13 & -2.59 & 4.91 & 0.14 & 15 \\
\hline $\mathrm{CoI}$ & 0.03 & 2.41 & 0.30 & 3 & 0.14 & 2.44 & 0.29 & 3 & -0.04 & 2.27 & 0.29 & 2 & $\cdots$ & $\cdots$ & $\cdots$ & \\
\hline NiI & 0.00 & 3.61 & 0.31 & 11 & 0.13 & 3.66 & 0.32 & 5 & 0.22 & 3.75 & 0.43 & 5 & 0.04 & 3.68 & 0.33 & 6 \\
\hline $\mathrm{CuI}$ & $<-0.5$ & $<1.28$ & $\ldots$ & 1 & $<0.5$ & $<2.00$ & $\ldots$ & 1 & $<0.0$ & $<1.50$ & $\ldots$ & 1 & -0.20 & 1.41 & $\ldots$ & 1 \\
\hline $\mathrm{ZnI}$ & -0.08 & 1.87 & $\cdots$ & 1 & 0.33 & 2.20 & 0.18 & 2 & 0.19 & 2.06 & $\cdots$ & 1 & 0.22 & 2.20 & 0.22 & 2 \\
\hline SrII & 0.09 & 0.35 & 0.33 & 2 & -0.26 & -0.08 & 0.32 & 2 & 0.04 & 0.22 & 0.31 & 2 & -0.03 & 0.27 & 0.31 & 2 \\
\hline YII & -0.20 & -0.60 & 0.26 & 5 & $<-0.3$ & $<-0.78$ & $\cdots$ & & -0.28 & -0.76 & 0.22 & 3 & -0.05 & -0.42 & 0.25 & 2 \\
\hline ZrII & 0.05 & 0.02 & 0.26 & 3 & 0.00 & -0.11 & $\cdots$ & 1 & -0.04 & -0.15 & 0.27 & 2 & 0.29 & 0.29 & 0.26 & 2 \\
\hline BaII & 0.11 & -0.32 & 0.32 & 4 & -0.20 & -0.71 & 0.38 & 4 & -0.05 & -0.56 & 0.24 & 4 & 0.14 & -0.26 & 0.34 & 4 \\
\hline LaII & 0.21 & -1.30 & 0.23 & 2 & 0.25 & -1.34 & $\cdots$ & 1 & 0.13 & -1.46 & 0.23 & 2 & 0.16 & -1.32 & 0.26 & 2 \\
\hline CeII & 0.32 & -0.71 & 0.29 & 4 & $<1.0$ & $<-0.11$ & $\cdots$ & & $<0.30$ & $<-0.81$ & $\cdots$ & & $<0.3$ & $<-0.70$ & $\cdots$ & \\
\hline PrII & 0.61 & -1.28 & $\cdots$ & & $<1.0$ & $<-0.97$ & $\cdots$ & & $<0.70$ & $<-1.27$ & $\cdots$ & & 0.50 & -1.36 & 0.23 & 3 \\
\hline NdII & 0.27 & -0.92 & 0.24 & 8 & 0.69 & -0.58 & 0.23 & 3 & 0.39 & -0.88 & 0.33 & 4 & 0.33 & -0.83 & 0.29 & 6 \\
\hline SmII & $<0.50$ & $<-1.15$ & $\cdots$ & & $<0.8$ & $<-0.93$ & $\cdots$ & & $<0.70$ & $<-1.03$ & $\cdots$ & & $<0.5$ & $<-1.12$ & $\cdots$ & \\
\hline EuII & 0.49 & -1.61 & 0.21 & 2 & 0.44 & -1.73 & 0.29 & 2 & 0.56 & -1.61 & 0.22 & 3 & 0.22 & -1.84 & 0.21 & 3 \\
\hline GdII & $<0.70$ & $<-0.84$ & $\ldots$ & & $<1.3$ & $<-0.32$ & $\ldots$ & & $<1.00$ & $<-0.62$ & $\ldots$ & & $<0.8$ & $<-0.71$ & $\ldots$ & \\
\hline DyII & 0.59 & -0.92 & 0.24 & 3 & $<0.7$ & $<-0.89$ & $\cdots$ & & $<1.00$ & $<-0.59$ & $\cdots$ & & 0.70 & -0.78 & 0.29 & 3 \\
\hline ErII & 0.50 & -1.19 & $\cdots$ & 1 & $<1.0$ & $<-0.77$ & $\ldots$ & & $<0.60$ & $<-1.17$ & $\cdots$ & & $\cdots$ & $\ldots$ & $\ldots$ & \\
\hline
\end{tabular}

\section{Results}

\subsection{Non-neutron-capture Elements}

We derive abundances for 18 non-neutron-capture elements from $\mathrm{C}$ to $\mathrm{Zn}$, including a range of $\alpha$-elements $(\mathrm{Mg}, \mathrm{Si}, \mathrm{Ca}, \mathrm{Ti})$ and iron peak elements $(\mathrm{Cr}, \mathrm{Mn}, \mathrm{Fe}, \mathrm{Co}, \mathrm{Ni})$. Figure 4 shows the abundances of 15 of these elements as a function of $[\mathrm{Fe} / \mathrm{H}]$ compared to stars in other ultra-faint dwarf galaxies and stars in the Milky Way halo. When considering the sample of all five stars, a range of metallicity $([\mathrm{Fe} / \mathrm{H}])$ is observed as well as the 

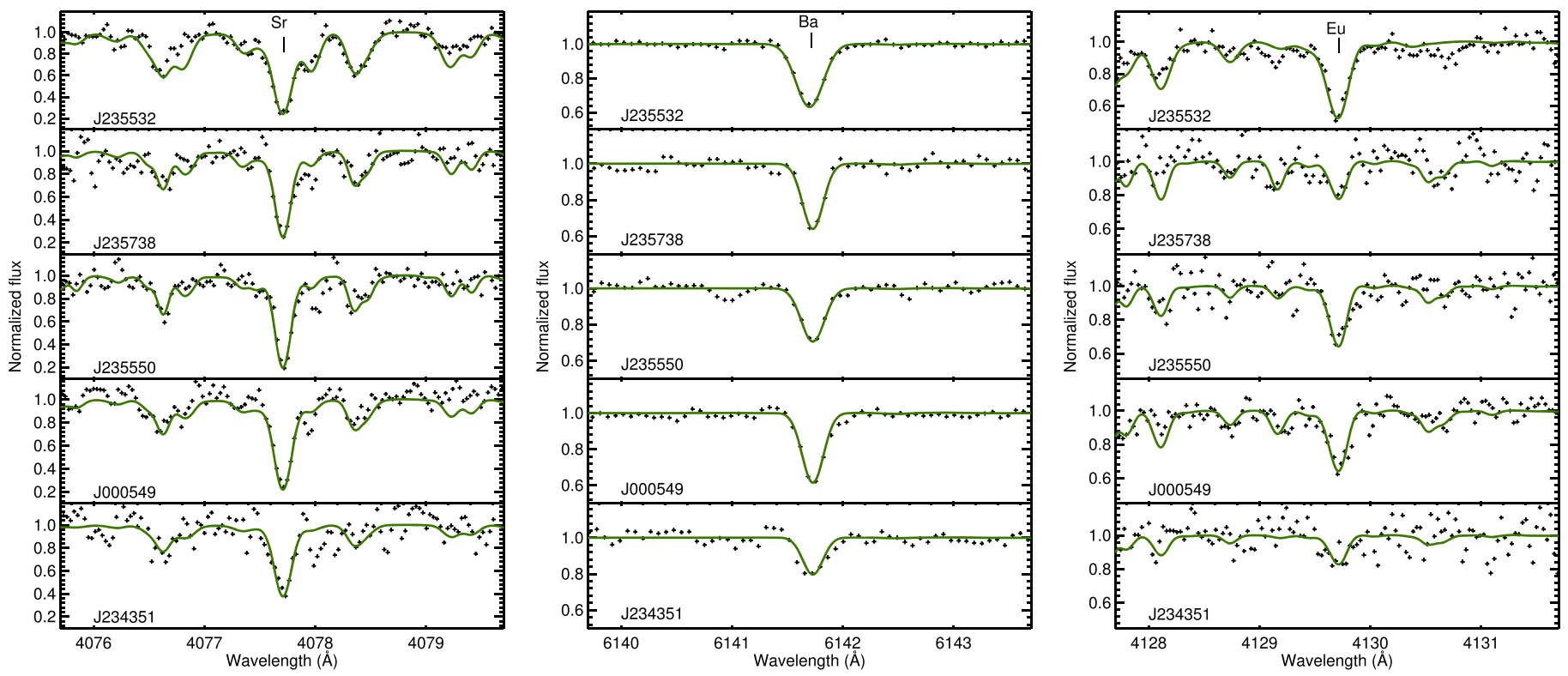

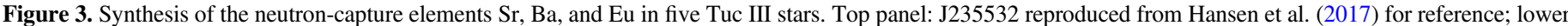
four panels: J235738 (top), J235550 (middle top), J000549 (middle bottom), and J234351 (bottom), this work.

Table 5

Uncertainties from Stellar Parameters for DES J00549

\begin{tabular}{|c|c|c|c|c|c|}
\hline Element & $\Delta T_{\text {eff }}$ & $\Delta \log g$ & $\Delta V_{\text {mic }}$ & $\Delta[\mathrm{Fe} / \mathrm{H}]$ & $\overline{\text { Total }}$ \\
\hline $\mathrm{C}$ & 0.16 & 0.02 & 0.1 & 0.17 & 0.25 \\
\hline $\mathrm{Na}$ & 0.24 & 0.12 & 0.1 & 0.17 & 0.33 \\
\hline Mg I & 0.14 & 0.05 & 0.05 & 0.17 & 0.23 \\
\hline $\mathrm{Al} \mathrm{I}$ & 0.27 & 0.17 & 0.04 & 0.17 & 0.36 \\
\hline Si I & 0.23 & 0.03 & 0.12 & 0.17 & 0.31 \\
\hline K I & 0.16 & 0.03 & 0.07 & 0.17 & 0.25 \\
\hline $\mathrm{Ca} \mathrm{I}$ & 0.12 & 0.03 & 0.07 & 0.17 & 0.22 \\
\hline Sc I & 0.07 & 0.08 & 0.18 & 0.17 & 0.27 \\
\hline Ti I & 0.22 & 0.04 & 0.04 & 0.17 & 0.28 \\
\hline Ti II & 0.06 & 0.08 & 0.13 & 0.17 & 0.24 \\
\hline V I & 0.21 & 0 & 0.02 & 0.17 & 0.27 \\
\hline V II & 0.07 & 0.1 & 0 & 0.17 & 0.21 \\
\hline Cr I & 0.2 & 0.04 & 0.08 & 0.17 & 0.28 \\
\hline $\mathrm{Cr}$ II & 0.03 & 0.1 & 0.02 & 0.17 & 0.2 \\
\hline Mn I & 0.15 & 0.02 & 0.05 & 0.17 & 0.23 \\
\hline Co I & 0.2 & 0.1 & 0.05 & 0.17 & 0.29 \\
\hline $\mathrm{Ni} I$ & 0.17 & 0.03 & 0.07 & 0.17 & 0.25 \\
\hline $\mathrm{Zn} \mathrm{I}$ & 0.06 & 0.04 & 0 & 0.17 & 0.18 \\
\hline $\mathrm{Sr}$ & 0.15 & 0.05 & 0.2 & 0.17 & 0.31 \\
\hline $\mathrm{Y}$ & 0.06 & 0.1 & 0.02 & 0.17 & 0.21 \\
\hline $\mathrm{Zr}$ & 0.2 & 0 & 0.05 & 0.17 & 0.26 \\
\hline $\mathrm{Ba}$ & 0.09 & 0.07 & 0.01 & 0.17 & 0.2 \\
\hline $\mathrm{La}$ & 0.12 & 0.1 & 0 & 0.17 & 0.23 \\
\hline $\mathrm{Ce}$ & 0.1 & 0.1 & 0.01 & 0.17 & 0.22 \\
\hline $\operatorname{Pr}$ & 0.09 & 0.05 & 0.06 & 0.17 & 0.21 \\
\hline $\mathrm{Nd}$ & 0.11 & 0.09 & 0.02 & 0.17 & 0.22 \\
\hline $\mathrm{Eu}$ & 0.07 & 0.08 & 0.07 & 0.17 & 0.21 \\
\hline Dy & 0.13 & 0.09 & 0 & 0.17 & 0.23 \\
\hline $\mathrm{Er}$ & 0.11 & 0.13 & 0.07 & 0.17 & 0.25 \\
\hline
\end{tabular}

expected trend in $\alpha$-elements, i.e., that lower metallicity stars in the stellar population have higher $\alpha$ abundances because they were formed at a time at which stellar nucleosynthesis was dominated by $\mathrm{SNe}$ II, compared to the more metal-rich stars that are formed later after the stellar population has been polluted by SN Ia explosions (Tinsley 1979). This $\alpha$ "knee" is observed in the elements $\mathrm{Ca}$ and $\mathrm{Ti}$; see Figure 4.

None of the stars can be classified as carbon-enhanced metalpoor stars (CEMP; $[\mathrm{C} / \mathrm{Fe}]>0.7)$.

\subsection{Neutron-capture Elements}

We derive abundances or upper limits for 11 neutron-capture elements from $\mathrm{Sr}$ to Er. Figure 5 shows abundances of the neutron-capture elements $\mathrm{Sr}, \mathrm{Ba}$, and $\mathrm{Eu}$ compared to stars in other ultra-faint dwarf galaxies and stars in the Milky Way halo.

Figure 6 shows the neutron-capture element abundance pattern for each of the stars compared to the solar system $s$ - and $r$-process abundance pattern from Simmerer et al. (2004). For each star the solar pattern has been scaled to the average residual between the star and the Sun for elements with abundances (not upper limits) from $\mathrm{Ba}$ to Er. It is clear from Figure 6 that the neutron-capture abundance pattern in four of the stars is better matched by the solar system $r$-process abundance pattern than the $s$-process pattern. J234351, with abundances measured for only six neutron-capture elements, is the exception. Neither the solar system $r$-nor $s$-process pattern matches the derived abundances for this star well. Note, however, that this star's spectrum has the lowest signal-to-noise of those studied here.

We adopt the Hansen et al. (2017) definition of $r$-process enhanced stars: $r$-I stars are defined to have $0.3<[\mathrm{Eu} / \mathrm{Fe}]<1$ and $[\mathrm{Eu} / \mathrm{Ba}]>0.4$, while $r$-II stars have $[\mathrm{Eu} / \mathrm{Fe}]>1$ and $[\mathrm{Eu} / \mathrm{Ba}]>0.4$, with the additional constraint on $[\mathrm{Eu} / \mathrm{Ba}]$ added to the traditional definition of $r$-process enhancement in order to ensure that the enhancement is entirely due to elemental production via the $r$-process, and is not confused by contributions from the $s$-process. Since Eu is nearly entirely produced in the $r$-process (94\%, Koch \& Edvardsson 2002) but $\mathrm{Ba}$ is produced primarily in the $s$-process $(85 \%$ according to 

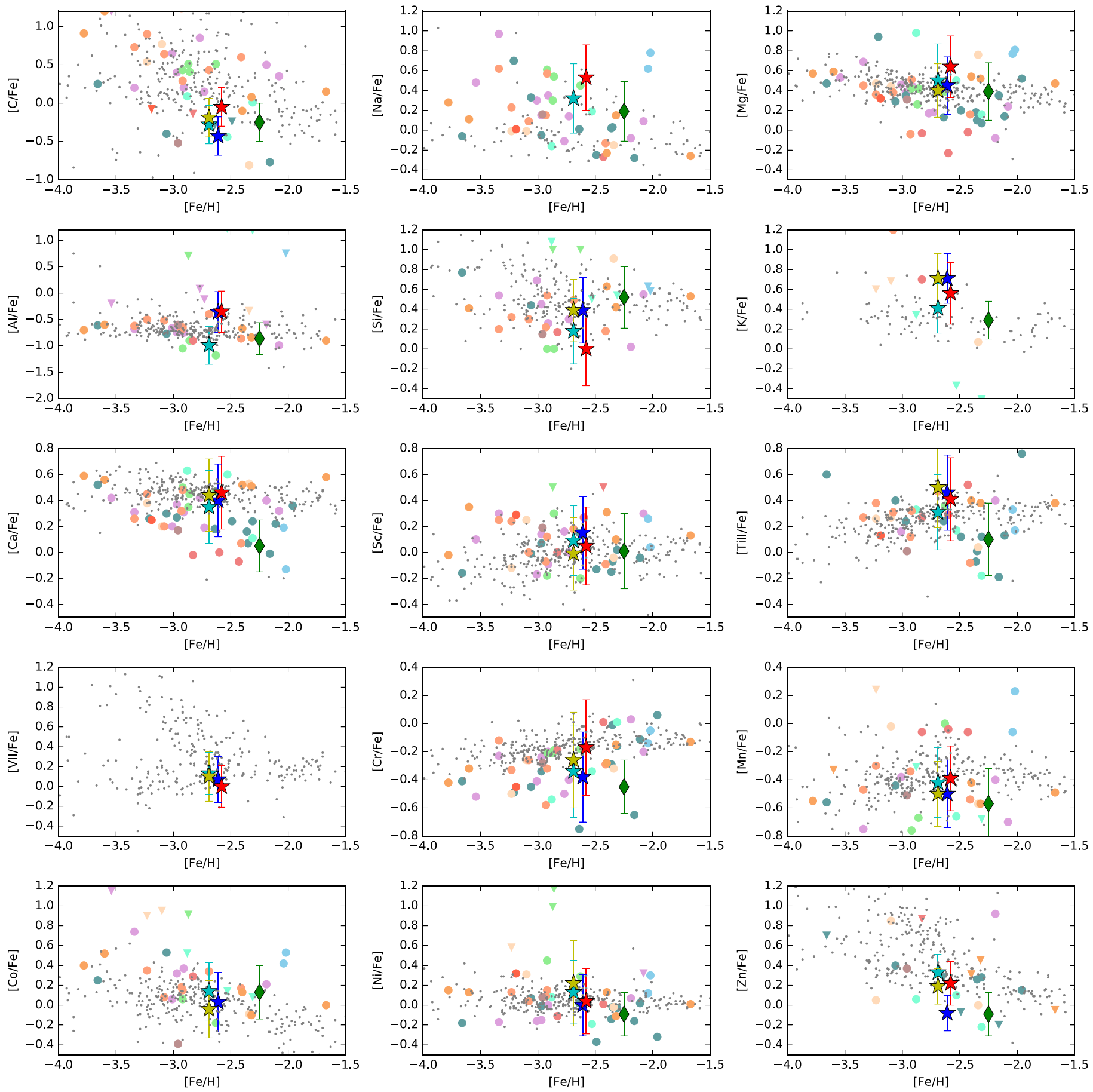

\begin{tabular}{|c|c|c|c|c|c|c|c|c|c|c|c|}
\hline 0 & Hor I & 0 & Tuc II & 0 & Boo II & 0 & Her & 0 & Leo IV & 0 & Segue 2 \\
\hline 0 & Ret II & 0 & Boo I & 0 & Com Ber & 0 & UMa II & 0 & Segue 1 & & \\
\hline
\end{tabular}

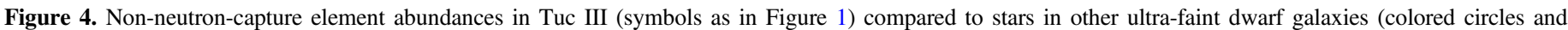

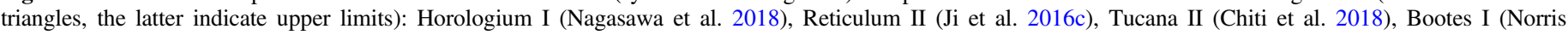

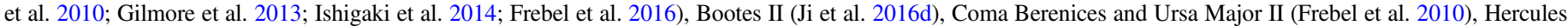

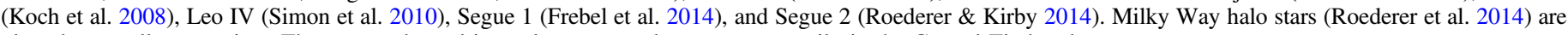
plotted as small gray points. The expected trend in $\alpha$-elements can be seen most easily in the $\mathrm{Ca}$ and $\mathrm{Ti}$ abundances.

Burris et al. 2000), the ratio of $\mathrm{Eu} / \mathrm{Ba}$ is often used to gauge whether the neutron-capture elements in a given star were produced primarily via the $s$ - or $r$-process.

Our measurements show that overall Tuc III is moderately enhanced in $r$-process elements: three of the four stars studied here are $r$-I stars having $0.3<[\mathrm{Eu} / \mathrm{Fe}]<1$, as is $\mathrm{J} 235532$, the Tuc III star previously studied by Hansen et al. (2017). Figure 7 shows the $[\mathrm{Eu} / \mathrm{H}]$ ratios as a function of metallicity for the five Tuc III stars along with $r$-process enhanced stars in other dwarf galaxies and in the Milky Way halo. 

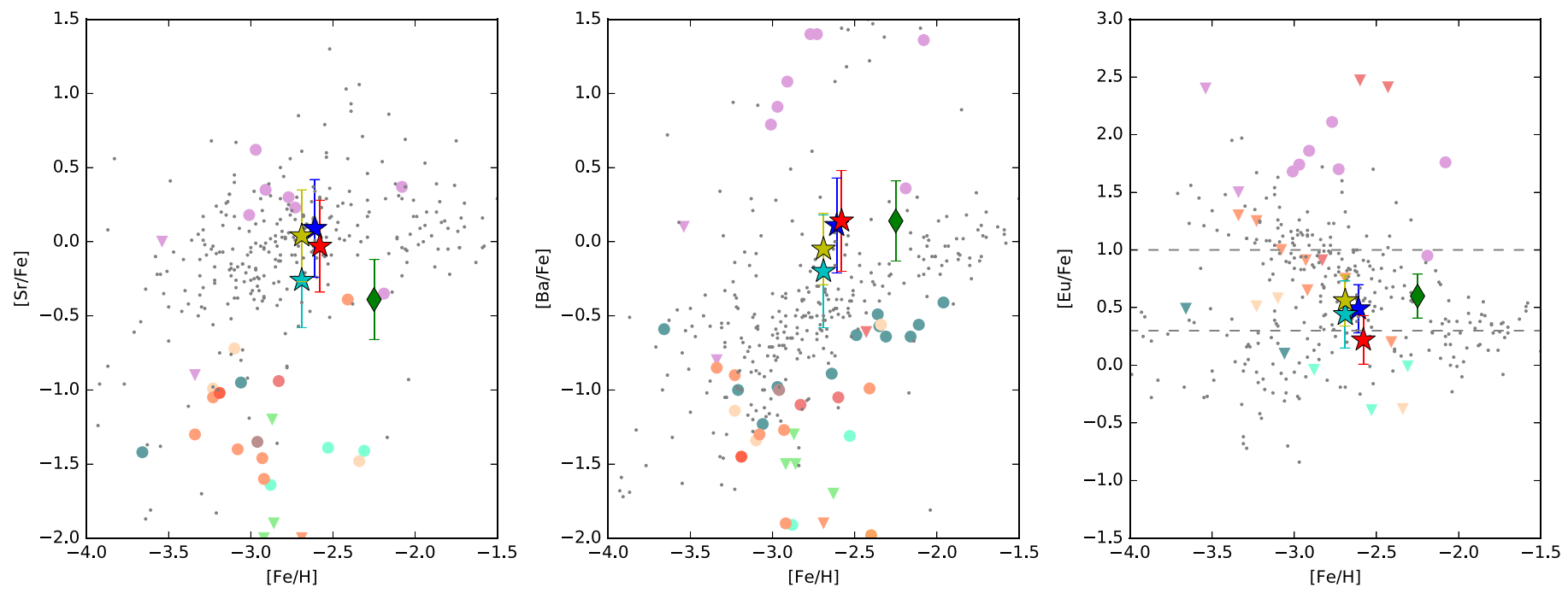

\begin{tabular}{|llllllllllll|}
\hline$\circ$ & Hor I & $\bigcirc$ & Tucll & $\circ$ & Booll & $\circ$ & Her & $\circ$ & LeolV & $\circ$ & Segue 2 \\
$\circ$ & Retll & $\circ$ & Bool & $\circ$ & ComBer & $\circ$ & UMall & $\circ$ & Segue 1 & & \\
\hline
\end{tabular}

Figure 5. Neutron-capture element abundances in Tuc III compared to other ultra-faint dwarf galaxies and Milky Way halo stars. Symbols as in Figure 4. Dashed lines in the right panel indicate the traditional definition of $r$-process enhanced stars: $r$-I stars have $0.3<$ [Eu/Fe] $<1 ; r$-II stars have [Eu/Fe] $>1$. Four of the five Tuc III stars lie within these boundaries and are classified as $r$-I stars; the fifth star (J235738) has error bars that cross the discriminator.

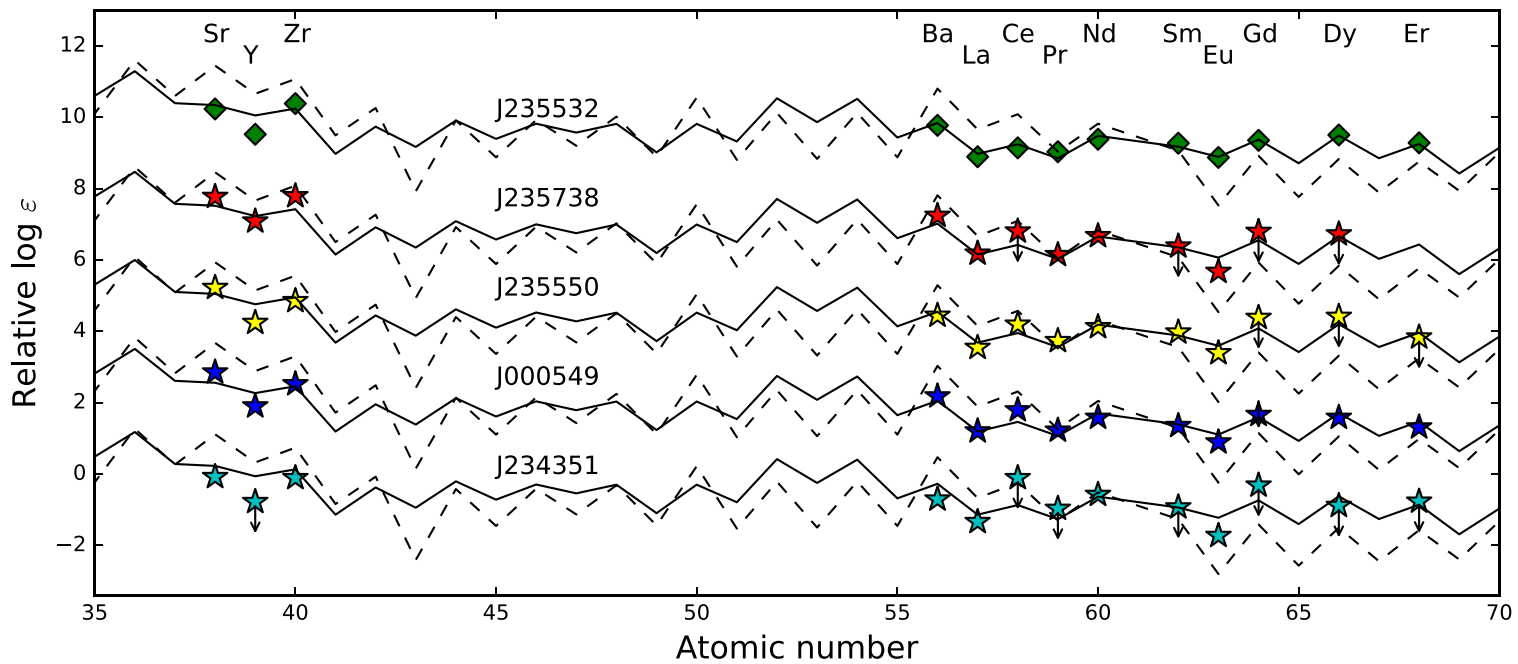

Figure 6. Absolute abundances of neutron-capture elements for our stars compared to scaled solar system $s$-process (dashed line) and $r$-process (solid line) abundance patterns, taken from Simmerer et al. (2004). A constant offset has been added to each star's abundances.

\subsection{Velocity Gradient}

Measured radial velocities of the stars studied here are consistent with Simon et al. (2017) and Li et al. (2018a) as shown in Figure 8. We collect all measured radial velocities for the five Tuc III stars in Table 6. We confirm the results of $\mathrm{Li}$ et al. (2018a), i.e., that there is a significant velocity gradient across the Tuc III system. We also see some evidence for velocity variations in individual stars, discussed in more detail in Section 5.3.

\section{Discussion}

Throughout this section we include the star DES J235532 studied by Hansen et al. (2017) in the discussion, increasing the sample size to five.

\subsection{The r-process Enhancement Event}

Tuc III is the second ultra-faint dwarf galaxy containing multiple stars enhanced in $r$-process elements. As discussed in Section 1, the first such galaxy, Ret II, is even more highly enhanced than Tuc III, i.e., many of the Ret II stars are $r$-II stars (see Figure 7). The fact that so many stars in a galaxy as small as Ret II share a common chemical pattern suggests that a single nucleosynthetic event must have occurred early in the history of the galaxy, polluting future generations of stars, and that most of the stars in the galaxy were impacted by the event.

The $r$-II stars in Ret II have an average enhancement in Eu of $[\mathrm{Eu} / \mathrm{H}] \sim-1$. Ji et al. (2016a) used this level and an estimated dilution gas mass, i.e., the mass of hydrogen gas that the $r$-process material is diluted into, of Ret II of $10^{6} M_{\odot}$ to argue that a binary neutron star merger was the most likely source of 


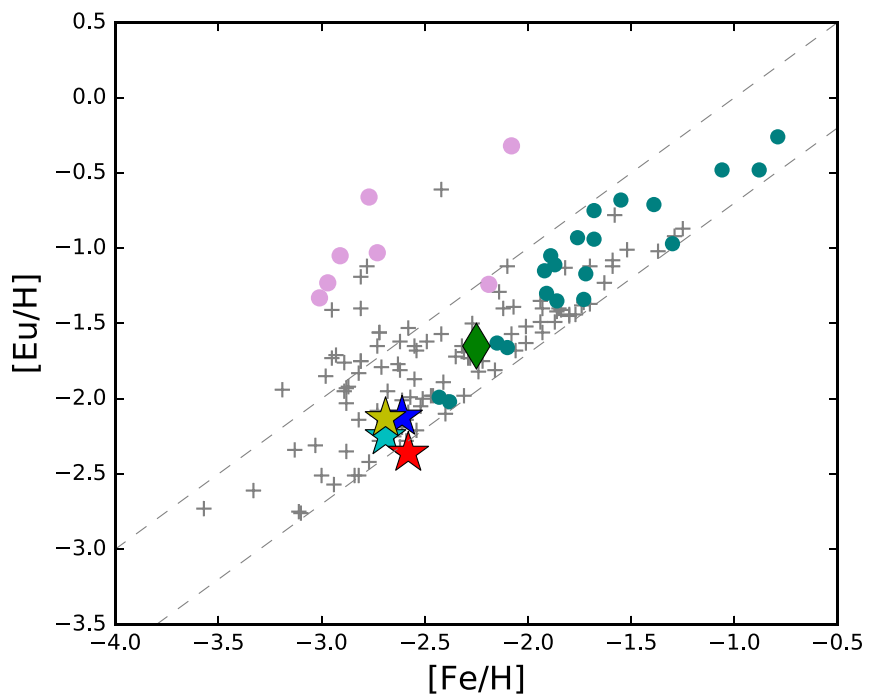

Figure 7. $[\mathrm{Eu} / \mathrm{H}]$ as a function of $[\mathrm{Fe} / \mathrm{H}]$ for the stars in Tuc III (symbols as in previous figures) compared to $r$-process enhanced stars in the halo (gray plusses) and other dwarf galaxies, including classical dwarfs (green dots; references given in Hansen et al. 2017) and Reticulum II (plum circles). Dashed lines show limits for $r$-I and $r$-II stars. The majority of stars in the halo and in other galaxies are not $r$-process enhanced; these would appear in the lower right and are not plotted here.

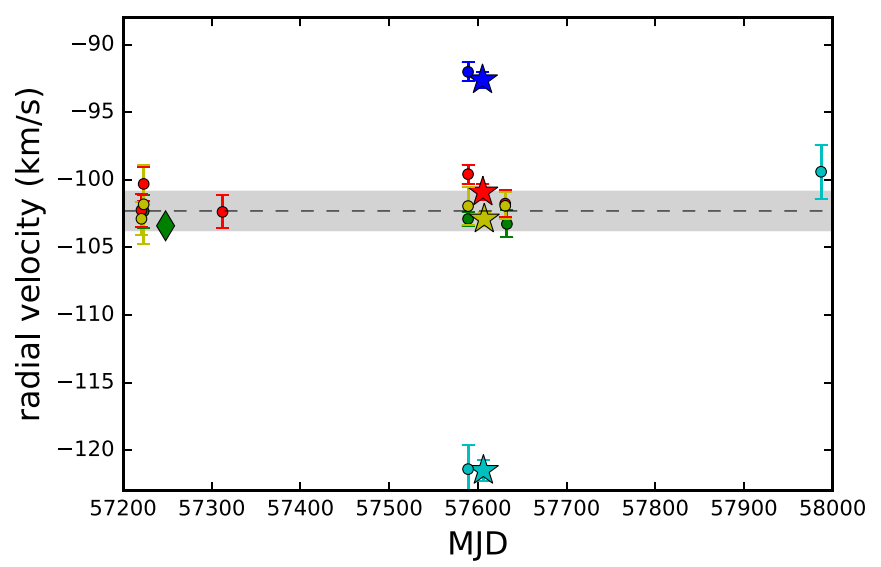

Figure 8. Radial velocities of the five stars as a function of time. Velocities measured by IMACS (Simon et al. 2017) and AAT (Li et al. 2018a) are marked as circles; symbols for the stars considered in this work are as in Figure 1. The dashed line and shaded region show the mean radial velocity and upper limit on the velocity dispersion of the Tuc III core stars as measured by Simon et al. (2017). The radial velocities of the tail stars confirm the velocity gradient measured in the AAT data. DES J234351 shows significant velocity variation and is likely in a binary system; DES J235738 may also be a binary.

the excess of Eu detected in Ret II as other sources would not have produced enough Eu to enhance the galaxy to the level detected. In Tuc III we find an average enhancement in Eu of $[\mathrm{Eu} / \mathrm{H}] \sim-2$. Assuming the same binary neutron star merger Eu ejecta mass as in Ji et al. (2016a), $10^{-4.5} M_{\odot}$, leads to a dilution gas mass of $\sim 2 \times 10^{6} M_{\odot}$, twice that of Ret II. If the $r$-process elements were produced via the same mechanism in Ret II and Tuc III, then either Tuc III had twice the gas mass to pollute or half the amount of $r$-process material. Current data do not distinguish between these possibilities, but we note that the ejecta mass from a neutron star merger could well vary from event to event (Côté et al. 2018). It is also worth noting that the stellar mass of Ret II is $(2.6 \pm 0.2) \times 10^{3} M_{\odot}$ (Bechtol et al. 2015), that of Tuc III is $(0.8 \pm 0.1) \times 10^{3} M_{\odot}$
Table 6

Radial Velocities from All Sources

\begin{tabular}{lccll}
\hline \hline Object Name & Date Observed $^{\mathrm{a}}$ & $v_{\text {hel }}$ & Error & References \\
\hline DES J235532 & 57248 & -103.4 & 0.3 & Hansen et al. (2017) \\
& 57223.3 & -102.32 & 1.23 & Simon et al. (2017) \\
& 57632.8 & -103.26 & 1.00 & Simon et al. (2017) \\
& $57589^{\mathrm{b}}$ & -102.89 & 0.51 & Li et al. (2018a) \\
\hline DES J235738 & 57605.69 & -100.9 & 0.8 & this work \\
& 57220.8 & -102.24 & 1.21 & Simon et al. (2017) \\
& 57223.3 & -100.3 & 1.27 & Simon et al. (2017) \\
& 57312.2 & -102.37 & 1.22 & Simon et al. (2017) \\
& 57630.9 & -101.76 & 1.00 & Simon et al. (2017) \\
& $57589^{\mathrm{b}}$ & -99.58 & 0.71 & Li et al. (2018a) \\
\hline DES J235550 & 57607.77 & -102.9 & 0.8 & this work \\
& 57220.8 & -102.88 & 1.21 & Simon et al. (2017) \\
& 57223.3 & -101.81 & 2.90 & Simon et al. (2017) \\
& 57630.9 & -101.92 & 1.00 & Simon et al. (2017) \\
& $57589^{\mathrm{b}}$ & -101.94 & 0.40 & Li et al. (2018a) \\
\hline DES J000549 & 57605.78 & -92.6 & 0.9 & this work \\
& $57589^{\mathrm{b}}$ & -92.01 & 0.70 & Li et al. (2018a) \\
\hline DES J234351 & 57606.82 & -121.5 & 1.1 & this work \\
& 57589 & -122.2 & 0.8 & Li et al. (2018a) \\
& 57987 & -99.4 & 1.4 & Li et al. (2018a) \\
\hline
\end{tabular}

Notes.

${ }^{a}$ Reported at the midpoint of the observation.

${ }^{\mathrm{b}}$ Average value over a 10 night observing run.

(Drlica-Wagner et al. 2015), and that of the Tuc III stream is $3.8 \times 10^{3} M_{\odot}$ (Shipp et al. 2018), so many possible dilution scenarios are plausible.

Sakari et al. (2018b) show that $r$-process enhanced stars in the Milky Way halo at a range of metallicities have nearly identical $r$-process patterns, matching the solar system $r$-process pattern, regardless of the level of $r$-process enhancement. Hansen et al. (2017) also found this to be true for the $r$-process enhanced stars detected in classical and ultra-faint dwarf galaxies. The implication of this result is that there must either be a single mechanism for $r$-process element production, or else every $r$-process production mechanism must produce identical abundance patterns. If the former, the most likely site of $r$-process enhancement is binary neutron star mergers, based on observational evidence to date. Furthermore, in galaxies with masses as low as the ultra-faint dwarfs discussed here, the star formation history must have proceeded in such a way that there was likely only one enrichment event, early in the history of the galaxy (e.g., Ojima et al. 2018). The $r$-process enhancement of Tuc III is consistent with this result, although with a different $r$-process element or dilution gas mass than in the case of Ret II.

Hansen et al. (2017) noted that with a sample of one star it is difficult to determine whether the source of enhancement in this galaxy is inside or outside the galaxy. With this larger sample of stars in both the core and the tidal tails, we can now claim that the abundance pattern of Tuc III, like that of Ret II, must be due to an enhancement event inside the galaxy. Since it appears that stars throughout the galaxy show similar levels of $r$-process enhancement, we can further state that this enhancement event must have occurred early in the history of the galaxy, thereby polluting the galaxy on large scales. 


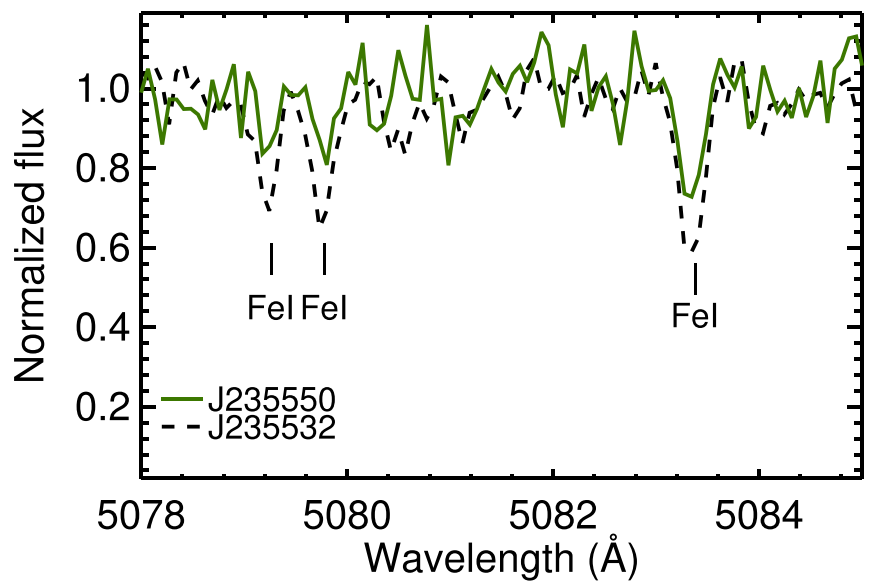

Figure 9. Comparison of the spectrum studied by Hansen et al. (2017; DES J235532) and of one of the stars in this work, DES J235550. The temperatures and surface gravities of these two stars are very similar; the metallicity difference between the two stars is apparent.

Safarzadeh \& Scannapieco (2017) used an adaptive mesh refinement cosmological simulation to show that the exact location of an enrichment event in small ultra-faint dwarf galaxies such as Ret II can have a large impact on the enrichment of all stars in the galaxy. In the case of Ret II, they compared the results of a binary neutron star merger located at the center of the galaxy compared to on the outskirts of the galaxy to show that the high levels of $r$-process enhancement seen in Ret II can only be explained if the event occurred very close to the center of the galaxy, and at a time at which the stars were still being formed. Another implication of the work of Safarzadeh \& Scannapieco (2017) is that a galaxy with lower levels of $r$-process enhancement, such as Tuc III, may have experienced a similar nucleosynthetic event, but that it was located at the edges of the galaxy. Such events occurring on the edges of the galaxy are not particularly unexpected, given the "kicks" binary neutron stars experience. Now that multiple stars in Tuc III have been shown to be moderately enhanced in $r$-process elements, a more detailed comparison can be made to this theoretical work.

\subsection{Galaxy or Globular Cluster?}

Both Simon et al. (2017) and Li et al. (2018a) considered in some detail the nature of Tuc III, because the low measured velocity dispersion $\left(0.1_{-0.1}^{+0.7} \mathrm{~km} \mathrm{~s}^{-1}\right)$ leads to speculation as to whether Tuc III was truly an ultra-faint dwarf galaxy or rather a globular cluster at birth. A possible reason for the low measured velocity dispersion, as discussed by Simon et al. (2017), is that stripping of the stars has lowered the velocity dispersion as Tuc III merges with the Galaxy. Since the velocity dispersion may not clearly determine the nature of Tuc III, we consider here several chemical aspects of the stellar population that may shed light on its origin.

The four stars studied here have a mean metallicity of $[\mathrm{Fe} / \mathrm{H}] \sim-2.64 \pm 0.15$, significantly lower than the star studied by Hansen et al. (2017) $([\mathrm{Fe} / \mathrm{H}] \sim-2.25)$. Figure 9 compares two of these spectra and demonstrates that Tuc III is not a monometallic system. Confirmation of Tuc III's metallicity dispersion using the five stars studied here provides further evidence that Tuc III is in fact a galaxy, because only galaxies, and not globular clusters, have gravitational wells deep enough to retain supernova ejecta, enabling the

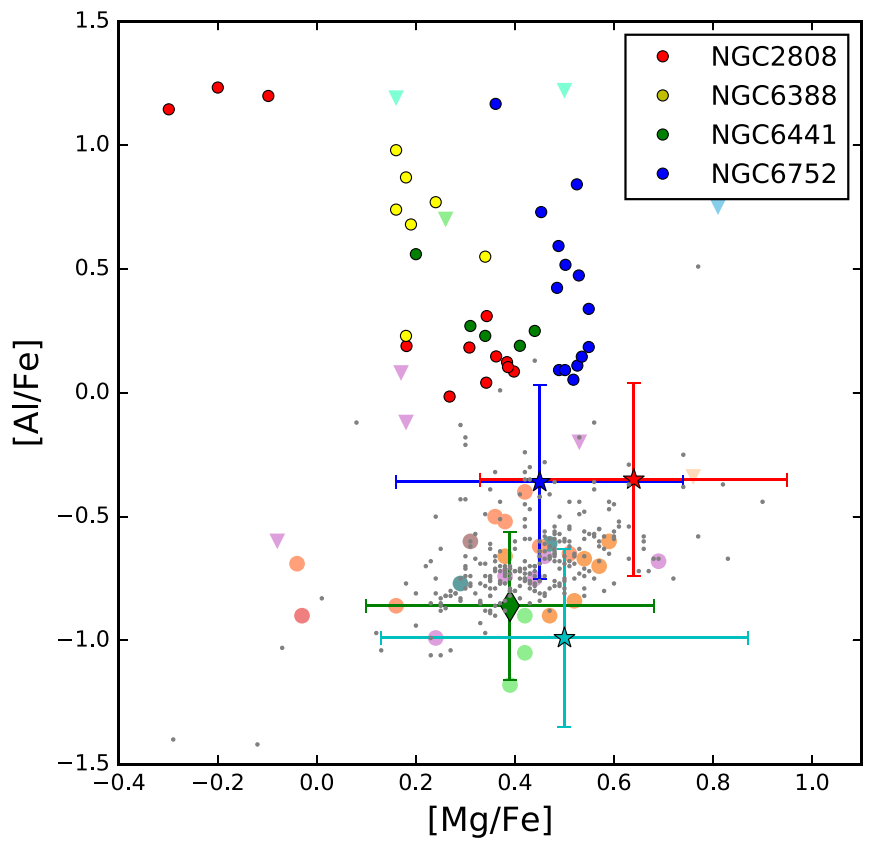

Figure 10. $[\mathrm{Al} / \mathrm{Fe}]$ as a function of $[\mathrm{Mg} / \mathrm{Fe}]$ for Tuc III stars compared to red giant stars in globular clusters NGC 2808 (Carretta et al. 2009), NGC 6388 (Carretta et al. 2007), NGC 6441 (Gratton et al. 2006), and NGC 6752 (Carretta et al. 2009), other ultra-faint dwarf galaxies (symbols as in previous figures), and stars in the halo (Roederer et al. 2014, gray points;). Tuc III does not appear to have a $\mathrm{Mg}-\mathrm{Al}$ anticorrelation, as would be expected if it were a globular cluster.

production of multiple generations of stars. The resulting range of metallicities observed in galaxies is the natural result of this extended formation (Tinsley 1979; Willman \& Strader 2012). We note that if we were to use the photometric temperature for DES J235532 we would derive a $\sim 0.2$ dex lower metallicity for this star. Consequently, using the photometric temperatures to consider the metallicities of these stars would result in no statistically significant metallicity range between the stars, weakening the evidence that Tuc III is a galaxy and not a globular cluster.

We further investigate the nature of Tuc III by considering the abundances of elements involved in proton-capture reactions, specifically $\mathrm{Mg}$ and $\mathrm{Al}$. The $\mathrm{Mg}-\mathrm{Al}$ anticorrelation that is observed in globular clusters is thought to be produced via pollution of second generation stars in the cluster by massive asymptotic giant branch stars at the end of their lives, particularly in massive or very metal-poor clusters (e.g., Carretta et al. 2009). Figure 10 compares the $\mathrm{Mg}$ and $\mathrm{Al}$ abundances of the Tuc III stars to red giant stars in four globular clusters that have been shown to have a strong $\mathrm{Mg}-\mathrm{Al}$ anticorrelation. The Tuc III stars studied here do not exhibit the proton burning trend.

Finally, while it is true that globular clusters generally have neutron-capture enhancement similar to that observed here (and very different from other ultra-faint dwarf galaxies, see Ji et al. 2018, for example), we do not feel that this commonality is enough to claim that Tuc III is more likely to be a globular cluster. Furthermore, Tuc III's average metallicity, $[\mathrm{Fe} / \mathrm{H}] \sim$ -2.49 ( $\mathrm{Li}$ et al. 2018a), would place it on the extreme low end of the distribution of globular cluster metallicities: the lowest metallicity globular cluster in the Harris $(1996,2010)$ catalog is NGC 7078 with $[\mathrm{Fe} / \mathrm{H}]=-2.37$. We therefore conclude that 
Tuc III is most likely an ultra-faint dwarf galaxy and not a globular cluster.

\subsection{Binarity}

Since all five of our stars were studied using multiple observations over a span of two years, we can use multiepoch radial velocities to search for reflex motion due to the stars being in an undetected binary system. In Figure 8 we compare the radial velocities measured in this work with those measured by Simon et al. (2017) and Li et al. (2018a). We see very good agreement between the measurements of three of the stars: DES J235532, DES J235550, and DES J000549 do not appear have variable radial velocities according to these measurements and are therefore unlikely to be binaries with periods $\lesssim 1 \mathrm{yr}$. Two other stars may have unseen binary companions: DES J234351 shows large radial velocity variation, and DES J235738 may also show small velocity variation.

DES J234351 is identified as a binary by Li et al. (2018a) as well, who note a $\Delta v \sim 20 \mathrm{~km} \mathrm{~s}^{-1}$ and exclude the star from further analysis of Tuc III's kinematics. Our higher precision velocities confirm the velocity shift between observations made in 2016 and 2017 and indicate that DES J234351 is very likely a binary system.

DES J235738 shows potential velocity variation with amplitude of $\geqslant 2.79 \mathrm{~km} \mathrm{~s}^{-1}$. This (weakly) suggests that this star may in fact be a binary and warrants further kinematic measurements. If DES J235738 is shown to be in a binary system, mass transfer from its companion could potentially explain its chemistry as well, because this star appears to have some $s$-process enhancement in addition to the $r$-process enhancement shared with the other stars, although a higher $\mathrm{S} / \mathrm{N}$ spectrum is needed to confirm this suggestion.

These are not the first binary stars discovered in an ultra-faint Milky Way satellite galaxy. Koch et al. (2014) measured velocities of one star in the Hercules dwarf galaxy over a two year baseline and concluded that it was in fact a binary system having a 135 day period, composed of a giant with a low-mass companion, likely a white dwarf. Despite the fact that mass transfer binaries can explain peculiar abundance patterns in some cases, no such binary scenario could be described by Koch et al. (2014) in the case of Hercules. Conversely, a binary star in Segue 1 does show signs of mass transfer (Frebel et al. 2014) through its high carbon abundance. Binary stars have also been detected via variable radial velocity signatures in Boo II (Ji et al. 2016d), Tri II (Kirby et al. 2017; Venn et al. 2017), Carina II (Li et al. 2018b), and Ret II (Simon et al. 2015; Minor et al. 2019).

\section{Conclusions}

We have presented chemical abundance measurements of four additional confirmed member stars in the Tuc III stellar system: two stars located in the core of the galaxy and two in the tidal tails. Together with the star studied by Hansen et al. (2017), the sample of five stars shows that Tuc III is moderately enhanced in $r$-process elements $(r$-I), shows the expected trend in $\alpha$-elements, and is not carbon enhanced. At least one, and possibly two, of the stars are likely to be binaries. The abundance patterns of these stars suggest that Tuc III is an ultra-faint dwarf galaxy and not a globular cluster.

As can be seen in Figure 1, there are more than 10 additional confirmed member stars of Tuc III that are bright enough to be studied in this way with today's largest telescopes and could be added to this sample: three blue horizontal branch stars and eight additional stars on the giant branch with $g<19$ (although not all of these may be true members, see Pace \& Li 2018). In the near term, study of these stars could increase the sample somewhat, until the next generation of telescopes enables the study of additional, fainter stars at high resolution.

Interestingly, two of the recently discovered southern hemisphere ultra-faint dwarf galaxies, Ret II and Tuc III, have now been shown to have multiple stars enhanced in $r$-process elements to a greater or lesser extent. The other DESdiscovered ultra-faint dwarfs that have been studied chemically to date, Tuc II (Ji et al. 2016b), Gru I (Ji et al. 2018), and Hor I (Nagasawa et al. 2018), do not show $r$-process enhancement. Additional ultra-faint dwarfs have member stars that are bright enough to be studied chemically, and may further add to the census of $r$-process enhanced galaxies. The reason that Ret II and Tuc III, and none of the other galaxies, have multiple $r$ process enhanced stars is as yet unknown, but may become clearer with study of additional stars in these and other galaxies.

The authors thank the referee for a careful reading of the manuscript and are grateful to A. McWilliam for insightful comments. T. T. Hansen and J. D. Simon acknowledge support from National Science Foundation grant AST-1714873.

Funding for the DES Projects has been provided by the U.S. Department of Energy, the U.S. National Science Foundation, the Ministry of Science and Education of Spain, the Science and Technology Facilities Council of the United Kingdom, the Higher Education Funding Council for England, the National Center for Supercomputing Applications at the University of Illinois at Urbana-Champaign, the Kavli Institute of Cosmological Physics at the University of Chicago, the Center for Cosmology and Astro-Particle Physics at the Ohio State University, the Mitchell Institute for Fundamental Physics and Astronomy at Texas A\&M University, Financiadora de Estudos e Projetos, Fundação Carlos Chagas Filho de Amparo à Pesquisa do Estado do Rio de Janeiro, Conselho Nacional de Desenvolvimento Científico e Tecnológico and the Ministério da Ciência, Tecnologia e Inovação, the Deutsche Forschungsgemeinschaft and the Collaborating Institutions in the Dark Energy Survey.

The Collaborating Institutions are Argonne National Laboratory, the University of California at Santa Cruz, the University of Cambridge, Centro de Investigaciones Energéticas, Medioambientales y Tecnológicas-Madrid, the University of Chicago, University College London, the DES-Brazil Consortium, the University of Edinburgh, the Eidgenössische Technische Hochschule (ETH) Zürich, Fermi National Accelerator Laboratory, the University of Illinois at UrbanaChampaign, the Institut de Ciències de l'Espai (IEEC/CSIC), the Institut de Física d'Altes Energies, Lawrence Berkeley National Laboratory, the Ludwig-Maximilians Universität München and the associated Excellence Cluster Universe, the University of Michigan, the National Optical Astronomy Observatory, the University of Nottingham, The Ohio State University, the University of Pennsylvania, the University of Portsmouth, SLAC National Accelerator Laboratory, Stanford University, the University of Sussex, Texas A\&M University, and the OzDES Membership Consortium. 
Based in part on observations at Cerro Tololo InterAmerican Observatory, National Optical Astronomy Observatory, which is operated by the Association of Universities for Research in Astronomy (AURA) under a cooperative agreement with the National Science Foundation.

The DES data management system is supported by the National Science Foundation under grant Nos. AST-1138766 and AST-1536171. The DES participants from Spanish institutions are partially supported by MINECO under grants AYA2015-71825, ESP2015-66861, FPA2015-68048, SEV2016-0588, SEV-2016-0597, and MDM-2015-0509, some of which include ERDF funds from the European Union. IFAE is partially funded by the CERCA program of the Generalitat de Catalunya. Research leading to these results has received funding from the European Research Council under the European Union's Seventh Framework Program (FP7/20072013) including ERC grant agreements 240672, 291329, and 306478. We acknowledge support from the Australian Research Council Centre of Excellence for All-sky Astrophysics (CAASTRO), through project number CE110001020, and the Brazilian Instituto Nacional de Ciência e Tecnologia (INCT) e-Universe (CNPq grant 465376/2014-2).

This manuscript has been authored by Fermi Research Alliance, LLC under Contract No. DE-AC02-07CH11359 with the U.S. Department of Energy, Office of Science, Office of High Energy Physics. The United States Government retains and the publisher, by accepting the article for publication, acknowledges that the United States Government retains a nonexclusive, paid-up, irrevocable, world-wide license to publish or reproduce the published form of this manuscript, or allow others to do so, for United States Government purposes.

\section{ORCID iDs}

J. L. Marshall ๑ https://orcid.org/0000-0003-0710-9474

T. Hansen (1) https://orcid.org/0000-0001-6154-8983

K. Kuehn (ㄷ) https://orcid.org/0000-0003-0120-0808

A. B. Pace (1) https://orcid.org/0000-0002-6021-8760

A. Palmese (i) https://orcid.org/0000-0002-6011-0530

L. Strigari (1) https://orcid.org/0000-0001-5672-6079

A. Drlica-Wagner (i) https://orcid.org/0000-0001-8251-933X

C. Lidman (1) https://orcid.org/0000-0003-1731-0497

D. Brooks (ㄷ) https://orcid.org/0000-0002-8458-5047

M. Carrasco Kind (1) https://orcid.org/0000-0002-4802-3194

J. García-Bellido (i) https://orcid.org/0000-0002-9370-8360

D. W. Gerdes (1) https://orcid.org/0000-0001-6942-2736

R. A. Gruendl (i) https://orcid.org/0000-0002-4588-6517

D. L. Hollowood (1) https://orcid.org/0000-0002-9369-4157

R. Miquel (i) https://orcid.org/0000-0002-6610-4836

M. Soares-Santos (i) https://orcid.org/0000-0001-6082-8529

G. Tarle (i) https://orcid.org/0000-0003-1704-0781

\section{References}

Abbott, B. P., Abbott, R., Abbott, T. D., et al. 2017, ApJL, 848, L12 Asplund, M., Grevesse, N., Sauval, A. J., \& Scott, P. 2009, ARA\&A, 47, 481 Barklem, P. S., Christlieb, N., Beers, T. C., et al. 2005, A\&A, 439, 129 Bechtol, K., Drlica-Wagner, A., Balbinot, E., et al. 2015, ApJ, 807, 50 Beers, T. C., \& Christlieb, N. 2005, ARA\&A, 43, 531

Bernstein, R., Shectman, S. A., Gunnels, S. M., et al. 2003, Proc. SPIE, 4841, 1694

Burbidge, E. M., Burbidge, G. R., Fowler, W. A., \& Hoyle, F. 1957, RvMP, 29,547

Burris, D. L., Pilachowski, C. A., Armandroff, T., et al. 2000, ApJ, 544, 302 Carretta, E., Bragaglia, A., Gratton, R., et al. 2007, A\&A, 464, 967
Carretta, E., Bragaglia, A., Gratton, R., \& Lucatello, S. 2009, A\&A, 505, 139 Castelli, F., \& Kurucz, R. L. 2003, in IAU Symp. 210, Modelling of StellarAtmospheres, ed. N. Piskunov, W. W. Weiss, \& D. F. Gray (San Francisco, CA: ASP), A20

Chambers, K. C., Magnier, E. A., Metcalfe, N., et al. 2016, arXiv:1612.05560 Chiti, A., Simon, J. D., Frebel, A., et al. 2018, ApJ, 857, 74

Côté, B., Fryer, C. L., Belczynski, K., et al. 2018, ApJ, 855, 99 DES Collaboration 2005, arXiv:astro-ph/0510346

DES Collaboration 2018, ApJ, 239, 18

Dotter, A., Chaboyer, B., Jevremović, D., et al. 2008, ApJS, 178, 89 Drlica-Wagner, A., Bechtol, K., Allam, S., et al. 2016, ApJL, 833, L5 Drlica-Wagner, A., Bechtol, K., Rykoff, E. S., et al. 2015, ApJ, 813, 109 Drlica-Wagner, A., Sevilla-Noarbe, I., Rykoff, E. S., et al. 2018, ApJS, 235, 33

Drout, M., Piro, A. L., Shappee, B. J., et al. 2017, Sci, 358, 1570

Erkal, D., Li, T. S., Koposov, S., et al. 2018, MNRAS, 481, 3148

Frebel, A., Casey, A. R., Jacobson, H. R., \& Yu, Q. 2013, ApJ, 769, 57

Frebel, A., Norris, J. E., Gilmore, G., \& Wyse, R. F. G. 2016, ApJ, 826, 110

Frebel, A., Simon, J. D., Geha, M., \& Willman, B. 2010, ApJ, 708, 560

Frebel, A., Simon, J. D., \& Kirby, E. N. 2014, ApJ, 786, 74

Gilmore, G., Norris, J. E., Monaco, L., et al. 2013, ApJ, 763, 61

Gratton, R. G., Lucatello, S., Bragaglia, A., et al. 2006, A\&A, 4f55, 271

Hansen, T. T., Holmbeck, E. M., Beers, T. C., et al. 2018, ApJ, 858, 92

Hansen, T. T., Simon, J. D., Marshall, J. L., et al. 2017, ApJ, 838, 44

Harris, W. E. 1996, AJ, 112, 1487

Harris, W. E. 2010, arXiv:1012.3224

Ishigaki, M. N., Aoki, W., Arimoto, N., \& Okamoto, S. 2014, A\&A, 562, A146

Ji, A. P., Frebel, A., Chiti, A., \& Simon, J. D. 2016a, Natur, 531, 610

Ji, A. P., Frebel, A., Ezzeddine, R., \& Casey, A. R. 2016b, ApJ, 832, 3

Ji, A. P., Frebel, A., Simon, J. D., \& Chiti, A. 2016c, ApJ, 830, 93

Ji, A. P., Frebel, A., Simon, J. D., \& Geha, M. 2016d, ApJ, 817, 41

Ji, A. P., Simon, J. D., Frebel, A., et al. 2018, ApJ, 870, 83

Kelson, D. D. 2003, PASP, 115, 688

Kim, D., \& Jerjen, H. 2015, ApJL, 808, L39

Kim, D., Jerjen, H., Milone, A. P., et al. 2015, ApJ, 803, 63

Kirby, E. N., Boylan-Kolchin, M., Cohen, J. G., et al. 2013, ApJ, 770, 16

Kirby, E. N., Cohen, J. G., Simon, J. D., et al. 2017, ApJ, 838, 83

Koch, A., \& Edvardsson, B. 2002, A\&A, 381, 500

Koch, A., Hansen, T. T., Feltzing, S., et al. 2014, ApJ, 780, 91

Koch, A., McWilliam, A., Grebel, E. K., et al. 2008, ApJL, 688, L13

Koposov, S. E., Belokurov, V., Torrealba, G., \& Evans, N. W. 2015, ApJ, 805,130

Li, T. S., Simon, J. D., Kuehn, K., et al. 2018a, ApJ, 866, 22

Li, T. S., Simon, J. D., Pace, A. B., et al. 2018b, ApJ, 857, 145

Luque, E., Pieres, A., Santiago, B., et al. 2017, MNRAS, 468, 97

Luque, E., Queiroz, A., Santiago, B., et al. 2016, MNRAS, 458, 603

Martin, N. F., Nidever, D. L., Besla, G., et al. 2015, ApJL, 804, L5

Mashonkina, L., Gehren, T., Travaglio, C., \& Borkova, T. 2003, A\&A, 397,275

Minor, Q. E., Pace, A. B., Marshall, J. L., \& Strigari, L. E. 2019, MNRAS, 487, 2961

Nagasawa, D. Q., Marshall, J. L., Li, T. S., et al. 2018, ApJ, 852, 99

Norris, J. E., Yong, D., Gilmore, G., \& Wyse, R. F. G. 2010, ApJ, 711, 350

Ojima, T., Ishimaru, Y., Wanajo, S., et al. 2018, ApJ, 865, 87

Pace, A. B., \& Li, T. S. 2018, ApJ, 875, 77

Ramírez, I., \& Meléndez, J. 2005, ApJ, 626, 465

Roederer, I. U., \& Kirby, E. N. 2014, MNRAS, 440, 2665

Roederer, I. U., Mateo, M., Bailey, J. I., III, et al. 2016, AJ, 151, 82

Roederer, I. U., Preston, G. W., Thompson, I. B., et al. 2014, AJ, 147, 136

Safarzadeh, M., \& Scannapieco, E. 2017, MNRAS, 471, 2088

Sakari, C. M., Placco, V. M., Farrell, E. M., et al. 2018a, ApJ, 868, 110

Sakari, C. M., Placco, V. M., Hansen, T., et al. 2018b, ApJL, 854, L20S

Shappee, B., Simon, J. D., Drout, M. R., et al. 2017, Sci, 358, 1574

Shipp, N., Drlica-Wagner, A., Balbinot, E., et al. 2018, ApJ, 862, 114

Simmerer, J., Sneden, C., Cowan, J. J., et al. 2004, ApJ, 617, 1091

Simon, J. D. 2018, ApJ, 863, 89

Simon, J. D., Drlica-Wagner, A., Li, T. S., et al. 2015, ApJ, 808, 95

Simon, J. D., Frebel, A., McWilliam, A., et al. 2010, ApJ, 716, 446

Simon, J. D., Li, T. S., Drlica-Wagner, A., et al. 2017, ApJ, 838, 11

Sneden, C. 1973, ApJ, 184, 839

Sobeck, J. S., Kraft, R. P., \& Sneden, C. 2011, AJ, 141, 175

Tinsley, B. 1979, ApJ, 229, 1046

Venn, K. A., Starkenburg, E., Malo, L., et al. 2017, MNRAS, 466, 3741

Walker, M. G., Mateo, M., Olszewski, E. W., et al. 2015, ApJ, 808, 108

Willman, B., \& Strader, J. 2012, AJ, 144, 76 